\title{
Paper Interfaces for Learning Geometry
}

\author{
Quentin Bonnard, Himanshu Verma, Frédéric Kaplan, and Pierre Dillenbourg \\ CRAFT, École Polytechnique Fédérale de Lausanne, \\ RLC D1 740, Station 20, 1015 Lausanne, Switzerland \\ \{quentin. bonnard, h.verma, frederic.kaplan, pierre.dillenbourg\}@epfl.ch
}

\begin{abstract}
Paper interfaces offer tremendous possibilities for geometry education in primary schools. Existing computer interfaces designed to learn geometry do not consider the integration of conventional school tools, which form the part of the curriculum. Moreover, most of computer tools are designed specifically for individual learning, some propose group activities, but most disregard classroom-level learning, thus impeding their adoption. We present an augmented reality based tabletop system with interface elements made of paper that addresses these issues. It integrates conventional geometry tools seamlessly into the activity and it enables group and classroom-level learning. In order to evaluate our system, we conducted an exploratory user study based on three learning activities: classifying quadrilaterals, discovering the protractor and describing angles. We observed how paper interfaces can be easily adopted into the traditional classroom practices.
\end{abstract}

Keywords: Paper interfaces, Sheets, Cards, Geometry learning, Tabletop

\section{Introduction}

Geometry education in primary schools is a domain ripe for exploiting the possibilities of computers, as they allow for an easy exploration of the problem space. However, there are some constraints which make it difficult to effectively utilize computers in a classroom scenario. Particularly, they do not cover the entire curriculum, which is based on pen and paper. For example, the only way for children to learn how to draw an arc is by using a physical compass.

Paper interfaces can prove to be an effective solution to this dilemma, as paper is already situated and integrated in the classroom environment and its practices. In addition, paper is cheap to produce, yet persistent and malleable to adapt to the dynamics of the classroom. As a computer interface it can transform into a dynamic display capable of computing and processing data. Besides these benefits of paper interfaces, paper has different properties and affordances depending upon its material, shape and size. Also, many interface metaphors such as cut-copy-paste, files and folders, check-boxes etc. are actually inspired by practices involving paper. Effective identification of these properties followed by a proper utilization, might render the paper interface intuitive for the users to interact. We hypothesize that geometry education in primary schools can greatly 
benefit from the use of paper interfaces and their characteristics. For example, folding is a natural embodiment of axial symmetries, cutting can be the physical counterpart of recomposing figures in order to compute their areas.

In this article, we present an augmented reality based tabletop system to facilitate geometry learning for primary school children. Our system incorporates a camera-projector device which is capable of projecting content over sheets and cards placed on a table top. We also present three exploratory user studies to study the influence and feasibility of using paper interfaces in primary schools. We report on the observations related to these user studies with three different geometry learning activities concerning shapes and angles.

\section{Related work}

The domain of paper interfaces is broad and not very well defined, just like the paper is used as an umbrella term for a variety of artefacts and practices. The archetype of using paper consists of writing with a pen (or a pencil) on a white rectangular sheet, but paper interfaces have been built for book reading [1], sticky notes [2], painting [3], presentation notes [4], trading cards [5], postcards [6] and even cover sheets [7]. However, in this section we would focus on the approaches addressing education, and start with the work related to the use of computers in geometry.

\subsection{Computers in Geometry Education}

Many researchers have tried to study the use of computers in geometry education involving software controlled by mouse and screen [8], augmented reality systems [9], or emulation of pen and paper [10]. Garcia et al. [8] identified that students appreciate the ability to repeat a geometrical construction (and playing it step-by-step) as allowed by a computer. Also, Dynamic Geometry Software (DGS) such as Cabri Géomètre [11], GeoGebra ${ }^{1}$ enables learners to explore the dynamic behavior of a geometrical construction, i.e. what moves and what remains fixed under given constraints. Straesser [12] explains how DGS opens new possibilities in geometry education, by enabling geometric constructions not easily possible with pen and paper. However, the use of WIMP interfaces in teaching involves the risk of spending more time to learn the software than learning geometry, as these interfaces are completely different from the typical geometry tools frequently used in classrooms such as compass, ruler etc. [13].

Augmented reality interfaces aim at making the interaction more natural by integrating virtual elements in the real world. For example, Kaufmann and his colleagues [14] addressed some of the shortcomings of learning spatial geometry on a mouse/screen/keyboard system: with head mounted displays, the manipulation of 3 dimensional objects is more direct, and they allow for face-to-face collaboration. Martín-Gutiérrez [15] and his colleagues designed an augmented

\footnotetext{
${ }^{1}$ www.geogebra.org
} 
book combined with a screen to develop spatial abilities in engineering students. They measured a positive impact on the spatial abilities, and the users found the system easy-to-use, attractive, and useful. Underkoffler and Ishii [9] made the reality augmenting device even less intrusive than head mounted displays or screens by using the so called $I / O$ bulbs to simulate optics. $I / O$ bulbs are camera/projector system above an interaction surface allowing students to manipulate tangible artefacts representing optical elements and see the effects on the trajectory of light.

Oviatt [10] and her colleagues bring forward this intent of making the interface as quiet as possible. They compared how student worked on geometrical problems using pen and paper, and interfaces approximating pen and paper with less and less exactitude: a smart pen using the microscopic pattern, a pen tablet, and a graphical tablet. They showed that the closer from the familiar work practice (i.e. pen and paper), the better is the performance.

To summarize, computers can add an essential dimension to geometry learning: dynamic information. However, existing educational interfaces for geometry are not adapted to classroom education, where paper prevails. Thus, paper interfaces can act as a bridge between computers and learning practices.

\subsection{Paper-Based Interfaces in Education}

We review the work related to paper interfaces in education based on the two aspects of paper that can be useful in the educational context. The first one, introduced by Wellner's seminal paper [16] on linking digital documents with their paper counterpart, presents paper as the support for working transparently on a digital document and its physical copy. This aspect is important for education, because it allows the researcher to study and extend the existing practice, in order to integrate the classroom more easily. Practices existing in the classroom that can be augmented include taking notes [17], reading textbooks [18], storytelling [19,20], or drawing schema [21].

The second aspect of paper useful for deployment in the classroom is its tangible aspect. It provides a cheap, easy way to attach virtual elements to reality. For example, Radu and MacIntyre [22] used cards for their tangible programming environment for pupils. Song and her colleagues used a cube covered by marked paper [23] to combined the advantages of digital and physical media. Millner and Resnick [24] even used a paper plate to prototype a steering wheel control, with printed buttons. Several frameworks [25-27] have already been proposed to study the design space of tangible interfaces [28, 29]. For example, Hornecker and Dünser [30] showed that pupils expect the system to match the physical properties of the tangible interface.

In both aspects, it is important to identify the properties of paper. Regarding the work practices related to paper, McGee [31] analyzed the established usages in order to list the properties that natural interfaces should have. In their literature review [32], Klemmer and Landay classified the other approaches based on whether they were using a book, a document, a table, or a printer (among other things). 
To sum-up, it has been identified that paper has two characteristics: it can be annotated, and it can be easily manipulated. These two features are associated to the established classroom practices that can be used to design intuitive paper interfaces. In this paper, we will investigate the most common forms allowing this: sheets and cards.

\section{System Used}

Our system for geometry education is built on the TinkerLamp [33], which is a tabletop environment developed at our lab. The TinkerLamp, shown in Figure 1 , incorporates a camera and a projector directed to the tabletop surface via a mirror, which extends the augmented surface. The augmented surface is of dimension $70 \times 40 \mathrm{~cm}$. The camera and projector are connected to an embedded computer, so that the interaction with the hardware is minimum for the end user: switch ON or OFF. It only requires to be plugged into an electric outlet.

We use fiducial markers similar to $\mathrm{ARTags}^{2}$ to identify and precisely track the various elements of the interface. Since the interface is projected from the top, it is possible to use interface elements (paper sheets and cards) as a projection surface in addition to the tabletop surface.

The different interface elements mainly consist of paper sheets and cards. The properties and behaviors of these interface elements are identified by the system using the fiducial markers printed over them. In addition to paper elements, we also use traditional geometry tools such as ruler and protractor as part of our system. We refer to this kind of interface as a scattered interface [34].

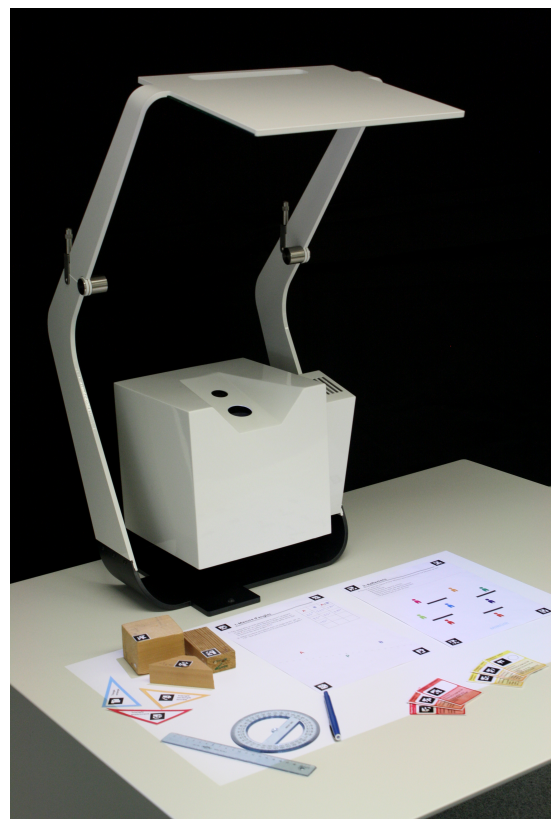

Fig. 1: Our camera-projector on a table, along with various types of objects which can be augmented: sheets, cards, tools and wooden blocks.

\section{Exploratory User Study}

In order to study the influence and potential of paper interfaces in geometry education for primary school pupils, we used our system to design three learning

\footnotetext{
$\overline{2}$ http://www $\cdot \operatorname{artag} \cdot$ net
} 
activities. These activities are based on cards and sheets, which are the two elements of our interface. Each activity was designed while keeping in mind the three circles of usability in the classroom - individual, group and classroom as examined by Dillenbourg et al. [35]. This was done in order to integrate the system well in the conventional classroom curriculum.

Our analysis is based on observational field notes made during the experiment, the videos from a panoramic camera placed under the lamp filming the pupils, and the snapshots of the interaction surface taken every second by the camera of the lamp. We logged the position of every fiducial marker with a time stamp, which allowed us to replay the interaction with the system, since the fiducial markers are the only input of the projected augmentation. This way, we could generate any additional log from the software. From the information collected, it would be possible to conduct more detailed analyses, however this will be the topic of future work.

\subsection{First Activity: Classifying Quadrilaterals}

We designed the first activity as a pedagogical script to introduce the classification of quadrilaterals (squares, rhombuses, trapezoids, etc.) as shown in Figure 2a. The script consists of sheets, four cards, and a set of quadrilateral cardboard shapes. Each of these elements has a fiducial marker to identify them and they were produced with a regular printer. The cardboard shapes were numbered, so that they could be referenced from the sheets.

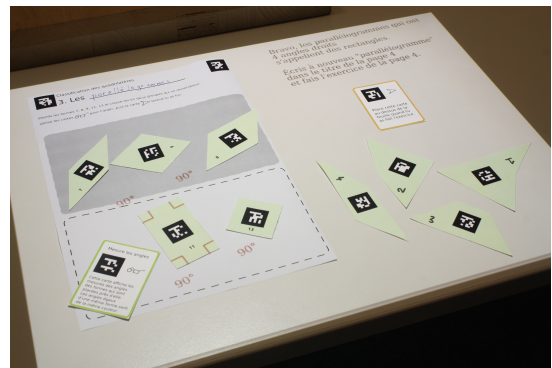

(a) The components of the activity about the classification of quadrilaterals: five cardboard quadrilaterals are classified into two groups on the instruction sheet, a card shows the measure of the angles of a rectangle, the feedback card displays the validation text.

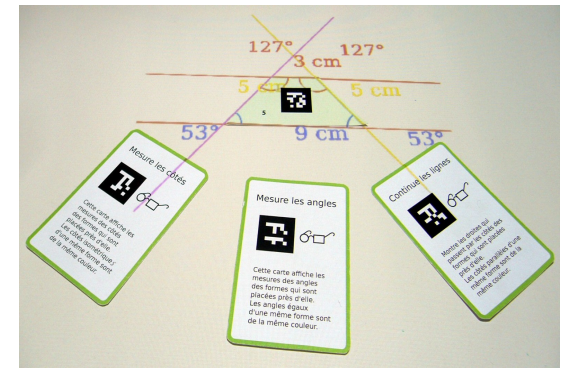

(b) Configuration of the tool cards into a test bench, where cardboard shapes (a trapezoid here) are brought to display all their characteristics.

Fig. 2: First Activity: Classifying Quadrilaterals

The sheets, carrying instructions, are shown in the left part of Figure 2a. They consists of a short instructional text and two areas (marked with different colors - gray and white) denoting two different classes of quadrilaterals. The text instructs the learner to use the three cards shown on Figure $2 \mathrm{~b}$ to find $\mathrm{a}$ 
common characteristic in a subset of shapes, and separate them into two classes. The cards have a small text describing their function. When a specific card is brought close to a shape, the system will display the given characteristic of the shape (such as side length, angle measures and parallel sides).

The learner is instructed to place a fourth card next to the current page once the shapes are placed in the classification areas (see the top right part of Figure 2a). If all shapes have not been placed in the areas, the learner will be reminded to do so. If the grouping is not the expected one, the learner will be invited to try again. If the grouping into areas is correct, the formulation of the answer will appear, e.g. "Good job! Quadrilaterals with a pair of parallel sides are called trapezoids". Feedbacks are intentionally trivial; the cards are not meant to replace teachers.

\section{Procedure and Discussion}

This activity was deployed at two occasions in schools with pupils in the age group of 7-10 years. On the first occasion, 13 pupils in groups of 2-3 individuals worked on the first sheet of the activity for 5 minutes. On the second occasion, the study was performed with 12 pupils (in groups of 3) who worked on the complete activity for 40 minutes. In both cases a short presentation of the system was given to the whole class. Hereafter, we present the observed usage of various interface elements while identifying their characteristic behaviors.

\section{Usage of the Cards}

- Cards are used as scaffolding. It is crucial that pupils learn how to measure using standard tools (ruler, protractor etc.). However, once these skills are mastered, the manual measurement can become menial and wastes time which can be utilized for the main topic of the lesson. In this regard, cards acted as scaffolds for skills that pupils have already mastered well (measuring side lengths). They also acted as scaffolds for skills that pupils did not master yet (drawing parallel lines), that was necessary to introduce another concept (trapezoids).

- Cards provided easy-to-use functionalities. We observed that pupils had no difficulty in using the cards, thanks to the printed self-description and their simple, easy to try functionalities. Cards were used in two ways: either they were brought close to the shape to display properties, or the shape was brought close to them.

- Cards allowed the composition of new functionalities. One group provided an interesting example of appropriation of the interface. They created a test bench by placing the tool cards together, and bringing the cardboard shapes in the common neighborhood of all the cards so as to show all the related information at once, as shown in Figure $2 \mathrm{~b}$.

\section{Usage of the Sheets}

- Sheets structured the activity in space. As opposed to the ephemeral workspaces that can emerge with cards as seen previously with the test bench, sheets 
predefined a necessary workspace i.e. the two areas corresponding to the groups in which the cardboard shapes are to be placed.

- Sheets structured the activity in time. The sequence of exercises is also predefined by the sheets. We note that this structure is flexible in a sense that if the teacher wants to skip an exercise in the software, it is as simple as skipping a page in the sheets.

\section{Usage of the Cardboard Shapes}

- Cardboard shapes are more concrete. Cardboard shapes can be replaced by cards with a textual description of shape or an illustration of the shape represented. However, the lower level of abstraction provided by the visual match between a geometrical shape and its corresponding cardboard representation, assists in reducing the cognitive effort to discover common points between them.

- Cardboard shapes are persistent. The cardboard shapes in this activity have an existence of their own, and not only in the context of our system. Since they are made of cardboard and not altered, they could be reused between two experiments.

\subsection{Second Activity: Discovering the Protractor}

We designed the second activity as an exploratory activity for pupils, in order to learn to use the protractor, after the introduction of angles in the classroom by the teacher. This activity incorporates a deck of cards of two kinds - two angle control cards and ten angle measure cards (see Figure 3a).

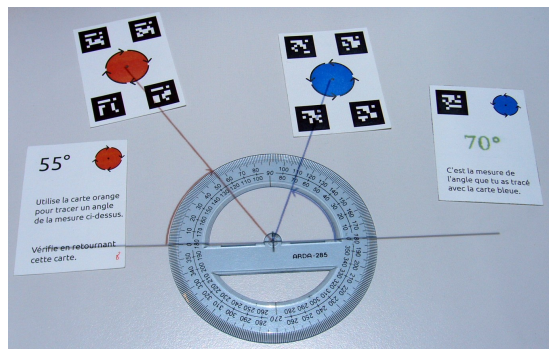

(a) The various elements of the task introducing angles: the two control cards and two of the angle measure. One is flipped and shows the measure of the angle constructed with the blue control card $\left(70^{\circ}\right)$.

Fig. 3: Second Activity: Discovering the Protractor

These cards can be divided into two groups based on the orange or a blue icon printed on them. These two colors indicate the direction of measurement of angles - orange cards correspond to clockwise measurement, whereas blue cards denote counter-clockwise measurement. This distinction had been identified during our 
collaboration with the teachers as the main difficulty when learning to use a protractor. When a control card is shown to the system, an angle appears, with its origin in the centre of the projection area, an extremity on the centre of the control card, and the other extremity fixed horizontally on the left or right side of the origin (the $\mathrm{X}$-axis), depending on whether the card is orange or blue (see Figure 3a).

Each angle measure card has a different angle value (in degrees) printed on them along with the instructions to construct an angle by using the corresponding control card. In order to check if the value of the produced angle (indicated on the angle measure card) is satisfying, the measure card is flipped and the current value of angle is displayed in a color depending on the degree of error (green for correct, yellow for close enough and red for otherwise).

\section{Procedure and Discussion}

This activity was conducted with 106 pupils (between 8-10 years) from 4 classes in a group of 2. Each group was required to go through 10 angle measure cards in 10 minutes, with individuals taking turns to measure angles. For the first 2 classes, the experimenters distributed the cards in a designated order one after the other. Whereas, for the other 2 classes the whole stack of cards was given to the group and no ordering was enforced. Also, the pupils were asked to take a pre-test and a post-test on paper where they were asked to identify and write down the angle measures next to a printed protractor as shown in Figure 3b. Next, we present our observations about how different interface elements were used.

\section{Usage of Cards}

- Cards materialize roles. This activity provides an example of group regulation via shared resources, as cards simply showed who was manipulating or checking. Also, time is regulated via the ownership of the control card. The pupils would try to homogenize the time each of them spends manipulating, as it is obvious who is doing all the work (i.e. having all the fun). This is beneficial since a lack of balance has been shown to reduce the benefits of learning in groups [36]. Similarly, having to share the control will encourage its negotiation, which has been shown to lead to greater learning gains [37].

- Cards materialize progress. Often, the measure cards were kept next to the pupil who managed to build the corresponding angle, acting as a trophy. Apart from the gain in engagement for the pupils, it is also a valuable help towards orchestration of the classroom, which refers to the teacher's responsibility to identify and manage the evolving learning opportunities and constraints, in real-time [35]. In this case, a teacher can easily get an instant summary of what each pupil did, and react accordingly.

- Cards materialize the mode. Cards also materialize even more ephemeral parts of the interaction, such as the current mode (building or checking). In this activity, it had a great implication on the engagement: all the groups preferred switching the feedback on and off for the sake of suspense rather 
than continuously displaying it. When we told them that they can also display the variations of measure of the angle being built, one pupil answered: "I'm hiding it to see if [the pupil manipulating the control card] manages to build the angle".

- The order of the cards did not matter. This activity revealed the fact that the order of the cards is not important, as pupils often selected the measure card they were more comfortable with. For example, a group skipped all the cards corresponding to clockwise angles. Out of the eight groups for which the order of the cards was not enforced, only two followed the designated sequence of cards. Two groups skipped angles of a given orientation (one built only clockwise angles while the other only counter-clockwise angles).

\section{Usage of Tools}

- Tools cannot be replaced. During the study, we realized the importance of using a real protractor and not a printed representation. The pre-test and post-test did not give any statistically significant results due to a ceiling effect, but yielded an interesting anecdote. During the pre-test, one of the pupil counted each increment within the angle to measure the graduation instead of reading the measure directly. During the activity, she correctly read the measure directly on the protractor. Again during the post-test, she counted the increments. She clearly did not match the printed graduation with the one on the real protractor.

\subsection{Third Activity: Describing Angles}

Whereas the second activity was designed to introduce the concept of measuring angles, the third activity regards describing and communicating angles. In order to communicate an angle to someone, the pupil has to describe the angle measure, direction of measurement (clockwise or anti-clockwise) as well as the most convenient reference for measuring this angle (which axis to choose). In this direction, the third activity was designed as a game to get rid of space junk, non-functional satellites that continue to orbit around Earth. We consider that there are 3 laser guns deployed at 3 locations around Earth capable of destroying space junk (see the right side of Figure 4). This activity also allows for the use of protractor during the problem solving task.

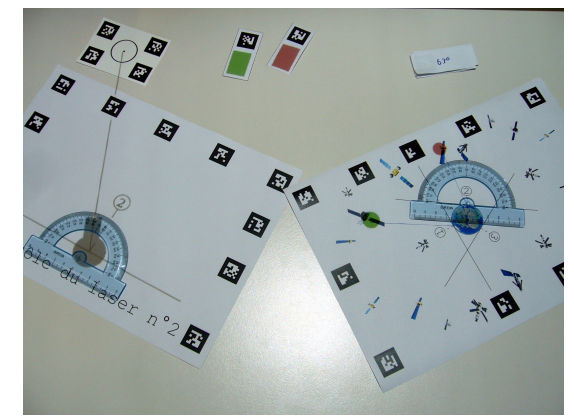

Fig. 4: The various elements of the problem using protractors.

We divide a group of 4 pupils into 2 collaborating teams (of 2 pupils) and call them observers and controllers, with a physical separation between them (see Figure 5). The observers have a sheet (right side of Figure 4) with the view of 


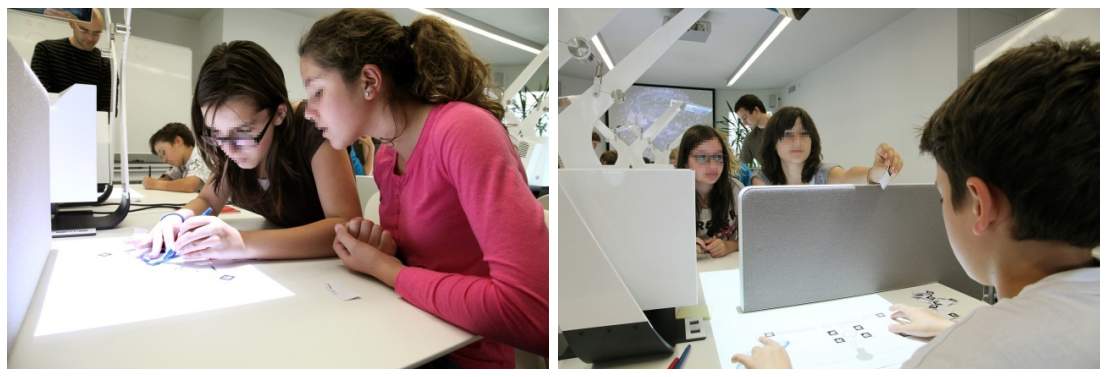

Fig. 5: The observer team measures the orientation to give to the laser (left), and communicates it to the controller team (right).

Earth along with all the satellites printed on them. Already destroyed satellites are highlighted in green and the next target in red. Also, the position of the three laser guns along with the baseline (axis) is also printed in the observer sheet. The observers are supposed to draw a line originating from one of the 3 laser guns to the target satellite (using a ruler). Next, they use the protractor to measure this angle with respect to the horizontal axis for this laser. Finally the observers have to describe this angle, direction of measurement and what laser gun to use to the controller team, by writing this information on a small piece of paper. This piece of paper is considered to be an ammunition for the laser gun.

The controllers are provided with 3 sheets corresponding to the 3 laser guns (see the left part of Figure 4). The controllers can change the inclination of the appropriate laser using the control card (similar to the one used in second activity). They reproduce the angle received from the observers using a protractor. Finally, the lasers can be activated by flipping this small paper received from the observers which contains a fiducial marker. Before firing, a yellow rectangle grows for 3 seconds over the ammunition (small paper), allowing to cancel the shot by flipping back or hiding the ammunition.

The trajectory of the laser is shown for 3 seconds on the sheets of controllers (laser gun) and observers (Earth view), with a fading blue line. If the satellite is hit, the ammunition turns green otherwise it turns red indicating a missed shot (see the centre top part of Figure 4). Each ammunition can only be used for a single shot, and the pupils are supplied with limited number of them, in order to avoid trial-and-error strategies.

\section{Procedure and Discussion}

We ran this activity on two occasions: once with 140 pupils from 7 classes and another time with 41 pupils from 2 classes. Groups of 4 pupils ( 2 observers and 2 controllers) were asked to complete this activity on a single system. Each group was given 25 minutes with this activity and they were asked to shoot as many satellites as they can. During the first study, we used 6 systems in a single room, while 2 systems were used in the second study. Next, we present our observations about the way sheets were used by groups during this activity.

\section{Usage of Sheets}


- The workspace of a sheet was a stable referential. Both observers and controllers placed their protractors on a sheet, which became a referential. All the groups but one kept the satellite view in the same orientation, even if it would have been easier to rotate the sheet before drawing the lines or measuring the angles.

- Progress was written on the sheet. While cards can act as ephemeral trophy, sheets durably store the progression with ink. The orchestration of a whole class was made a lot easier by the fact that the satellite view kept track of the intended trajectories in the form of lines between the location of the laser on Earth and the satellite. It helped to diagnose which part of the group (the observers or the controllers) was wrong in their measurement. The annotations on the ammunitions kept track of the progress of the group. The main difficulty in the activity is to establish a convention to describe and communicate an angle without seeing it. Giving the measurement was obvious, and the pupils would quickly realize that the origin of the shot (i.e. which laser to use) has to be communicated too. The more tricky part concerned the orientation of the angle. Since each shot has to be described on the ammunitions, it was easy to track when the pupils started to realize which information was needed.

- Sheets do not restrict expressiveness. When we explained the activity to the pupils, we intentionally remained vague on how to describe the angle, simply hinting them that there were several informations to provide. The angle measure and the laser gun to use were easily given as numbers. However, the pupils did not have an established convention for the orientation. This constructivist exploration paves the way for the teacher to explain the concept of clockwise and counter-clockwise measurement, since the need has been felt directly.

\section{Conclusion}

The tabletop system presented in this article was designed to facilitate geometry learning for primary school pupils. As existing classroom curriculum is based on paper and conventional geometry tools (ruler, compass, etc.), our system incorporates paper sheets and cards as the two main interface elements. We designed and conducted 3 exploratory user studies focusing on the different usages of sheets and cards, in order to study the impact and potential of paper interfaces in geometry learning. Our observations show very positive results regarding the adoption of paper interfaces by the pupils, as the use of sheets and cards was easily perceived and minimal effort was required to learn how to use the interface.

Our system takes into account the three circles of usability outlined by Dillenbourg et al. [35] - individual, group and classroom. On the individual level the pupils were highly engaged and participated actively in the activities, even in the classes that were less affected by the novelty effect in our subsequent visits. This is a success given that the activities revolved around using a (boring) protractor, or classifying quadrilaterals. On the group level, the system naturally 
promoted collaboration, allowing pupils to help each other and learn in teams. At the classroom level, the paper interface enabled the teacher to monitor the progress of teams and thus orchestrate the classroom activities accordingly. This aims at facilitating smooth integration and adoption of computers in the entire curriculum.

In addition, our observations regarding the characteristics of sheets and cards provided insights about the affordances of the different paper elements. On one hand, we observed that sheets are important for their content. Sheets were used to organize the discourse on two levels. On the first level, the layout on a single page encodes the order in which to read the various information and proceed with the activity. On the second level, several sheets can be organized together in a sequence (by stapling or binding), which enables us to implement several lessons or exercises similar to a book. As the trace of a pen is persistent over sheets, they can act as a permanent memory, which can be used as a way to trace the performance of pupils during a learning activity, or display publicly the progress within the group.

On the other hand, cards are mostly used as a physical body. The position of the card is usually relative, and bringing one close to another element allows to show additional properties. Further, the side of a card is another useful property; it can be flipped to control a binary value. In general, cards can materialize the reversible and ephemeral pieces of interaction according to rules. For example, the presence of a card on the table or next to a pupil indicated its role in the group.

We believe that careful identification of these characteristics of paper interface elements might provide crucial design guidelines towards the development of paper interfaces for education in general. The affordances of different paper elements (depending on the shape, size and material) render the interfaces easyto-use and highly intuitive.

In future, we would like to conduct a formal evaluation of the effects of paper interfaces on learning. Also, we would like to investigate the technological issues related to the predisposition of the system and to learning design. The aim would be to enable teachers to set up pedagogical experiences without assistance from researchers. This would naturally link the activities to specific mathematics learning theories.

\section{Acknowledgement}

The authors wish to sincerely thank the teachers for their precious collaboration, as well as Olivier Guédat for building the TinkerLamp; Michael Chablais, Chia-Jung Chan Fardel, and Carlos Sanchez Witt for their contribution to the activities during their internship.

\section{References}

1. Klemmer, S.R., Graham, J., Wolff, G.J., Landay, J.A.: Books with voices: paper transcripts as a physical interface to oral histories. In: CHI '03, New York, USA, 
ACM (2003) 89-96

2. Moran, T.P., Saund, E., Van Melle, W., Gujar, A.U., Fishkin, K.P., Harrison, B.L.: Design and technology for collaborage: collaborative collages of information on physical walls. In: UIST '99, New York, USA, ACM (1999) 197-206

3. Flagg, M., Rehg, J.: Projector-guided painting. In: UIST '06, ACM (2006) 235-244

4. Nelson, L., Ichimura, S., Pedersen, E.R., Adams, L.: Palette: a paper interface for giving presentations. In: CHI '99, New York, USA, ACM (1999) 354-361

5. Lam, A.H.T., Chow, K.C.H., Yau, E.H.H., Lyu, M.R.: Art: augmented reality table for interactive trading card game. In: VRCIA '06, New York, NY, USA, ACM (2006) 357-360

6. Cho, H., Jung, J., Cho, K., Seo, Y.H., Yang, H.S.: Ar postcard: the augmented reality system with a postcard. In: VRCIA '11, New York, USA, ACM (2011) 453-454

7. Hong, J., Price, M.N., Schilit, B.N., Golovchinsky, G.: Printertainment: printing with interactive cover sheets. In: CHI EA '99, New York, USA, ACM (1999) 240241

8. García, R., Quirós, J., Santos, R., González, S., Fernanz, S.: Interactive multimedia animation with Macromedia Flash in Descriptive Geometry teaching. Computers \& Education 49(3) (2007) 615-639

9. Underkoffler, J., Ishii, H.: Illuminating light: a casual optics workbench. In: CHI EA '99, New York, USA, ACM (1999) 5-6

10. Oviatt, S., Arthur, A., Brock, Y., Cohen, J.: Expressive pen-based interfaces for math education. In: CSCL, International Society of the Learning Sciences (2007) $573-582$

11. Laborde, C., Keitel, Ruthven, K.: The computer as part of the learning environment: the case of geometry. In: Learning from computers:Mathematics education and technology. Springer-Verlag (1993) 48-67+

12. Straesser, R.: Cabri-geometre: Does dynamic geometry software (dgs) change geometry and its teaching and learning? International Journal of Computers for Mathematical Learning 6(3) (2002) 319-333

13. Kortenkamp, U., Dohrmann, C.: User Interface Design For Dynamic Geometry Software. Acta Didactica Napocensia 3 (2010)

14. Kaufmann, H., Dünser, A.: Summary of usability evaluations of an educational augmented reality application. In: ICVR '07, Berlin, Heidelberg, Springer-Verlag (2007) 660-669

15. Martín-Gutiérrez, J., Luís Saorín, J., Contero, M., Alcaņiz, M., Pérez-López, D., Ortega, M.: Design and validation of an augmented book for spatial abilities development in engineering students. Computers \& Graphics 34(1) (2010) 77-91

16. Wellner, P.: Interacting with paper on the DigitalDesk. Communications of the ACM 36(7) (1993) 87-96

17. Malacria, S., Pietrzak, T., Tabard, A., Lecolinet, É.: U-note: capture the class and access it everywhere. Human-Computer Interaction-INTERACT 2011 (2011) 643-660

18. Asai, K., Kobayashi, H., Kondo, T.: Augmented instructions - a fusion of augmented reality and printed learning materials. In: ICALT, IEEE Computer Society (2005) 213-215

19. Portocarrero, E., Robert, D., Follmer, S., Chung, M.: The NeverEndingStorytellingMachine a platform for creative collaboration using a sketchbook and everyday objects. Proc. PaperComp'10 (2010) 
20. Koike, H., Sato, Y., Kobayashi, Y., Tobita, H., Kobayashi, M.: Interactive textbook and interactive venn diagram: natural and intuitive interfaces on augmented desk system. In: CHI '00, New York, USA, ACM (2000) 121-128

21. Lee, W., de Silva, R., Peterson, E., Calfee, R., Stahovich, T.: Newton's Pen: A pen-based tutoring system for statics. Computers \& Graphics 32(5) (2008) 511-524

22. Radu, I., MacIntyre, B.: Augmented-reality scratch: a children's authoring environment for augmented-reality experiences. In: IDC '09, New York, USA, ACM (2009) 210-213

23. Song, H., Guimbretière, F., Ambrose, M., Lostritto, C.: CubeExplorer: an evaluation of interaction techniques in architectural education. In: Proceedings of the 11th IFIP TC 13 international conference on Human-computer interaction-Volume Part II, Springer-Verlag (2007) 43-56

24. Millner, A., Resnick, M.: Tools for creating custom physical computer interfaces. Demonstration presented at Interaction Design and Children, Boulder, CO (2005)

25. Ullmer, B., Ishii, H.: Emerging frameworks for tangible user interfaces. IBM systems journal 39(3.4) (2000) 915-931

26. Ullmer, B., Ishii, H., Jacob, R.: Token+ constraint systems for tangible interaction with digital information. ACM Transactions on Computer-Human Interaction (TOCHI) 12(1) (2005) 81-118

27. Fishkin, K.: A taxonomy for and analysis of tangible interfaces. Personal and Ubiquitous Computing 8(5) (2004) 347-358

28. Fitzmaurice, G.: Graspable user interfaces. PhD thesis, Citeseer (1996)

29. Ishii, H., Ullmer, B.: Tangible bits: towards seamless interfaces between people, bits and atoms. In: CHI '97, New York, USA, ACM (1997) 234-241

30. Hornecker, E., Dünser, A.: Of pages and paddles: Children's expectations and mistaken interactions with physical-digital tools. Interacting with Computers 21(12) (2009) 95-107

31. Mcgee, D.R.: Augmenting environments with multimodal interaction. PhD thesis, Oregon Health \& Science University (2003) AAI3100651.

32. Klemmer, S.R., Landay, J.A.: Toolkit support for integrating physical and digital interactions. Human-Computer Interaction 24(3) (2009) 315-366

33. Zufferey, G., Jermann, P., Dillenbourg, P.: A tabletop learning environment for logistics assistants: activating teachers. In: Proceedings of the Third IASTED International Conference on Human Computer Interaction. HCI '08, Anaheim, CA, USA, ACTA Press (2008) 37-42

34. Cuendet, S., Bonnard, Q., Kaplan, F., Dillenbourg, P.: Paper interface design for classroom orchestration. In: Proceedings of the 2011 annual conference extended abstracts on Human factors in computing systems. CHI EA '11, New York, NY, USA, ACM (2011) 1993-1998

35. Dillenbourg, P., Zufferey, G., Alavi, H.S., Jermann, P., Do, L.H.S., Bonnard, Q., Cuendet, S., Kaplan, F.: Classroom orchestration : The third circle of usability. In: Connecting Computer-Supported Collaborative Learning to Policy and Practice: CSCL2011 Conference Proceedings. Volume I - Long Papers. Volume 1., International Society of the Learning Sciences (2011) 510-517

36. Cohen, E.: Restructuring the classroom: Conditions for productive small groups. Review of educational research 64(1) (1994) 1

37. Do-Lenh, S., Kaplan, F., Dillenbourg, P.: Paper-based concept map: the effects of tabletop on an expressive collaborative learning task. In: Proceedings of the 23rd British HCI Group Annual Conference on People and Computers: Celebrating People and Technology. BCS-HCI '09, Swinton, UK, UK, British Computer Society (2009) 149-158 